\title{
Article \\ Universal Dental Adhesives: Cost-Effectiveness and Duration of Use
}

\author{
Ayman A. Banjar (1) and Hani M. Nassar *(D)
}

Citation: Banjar, A.A.; Nassar, H.M. Universal Dental Adhesives:

Cost-Effectiveness and Duration of Use. Appl. Sci. 2022, 12, 487.

https://doi.org/10.3390/

app12010487

Academic Editor: Gabriele Cervino

Received: 27 November 2021

Accepted: 3 January 2022

Published: 4 January 2022

Publisher's Note: MDPI stays neutral with regard to jurisdictional claims in published maps and institutional affiliations.

Copyright: (c) 2022 by the authors. Licensee MDPI, Basel, Switzerland. This article is an open access article distributed under the terms and conditions of the Creative Commons Attribution (CC BY) license (https:// creativecommons.org/licenses/by/ $4.0 /)$.
Department of Restorative Dentistry, Faculty of Dentistry, King Abdulaziz University, Jeddah 21589, Saudi Arabia; aabanjar@kau.edu.sa

* Correspondence: hnassar@kau.edu.sa; Tel.: +966-12-695-2000
Abstract: The purpose of this study was to conduct a cost-effectiveness analysis (CEA) of different brands of universal dental adhesives used for composite restorations. Four adhesive brands were included: Single-Bond Universal (SB), Tetric N-Bond Universal VivaPen (TN), OptiBond All-In-One (OB), and G-Premio Bond (GP). Adhesives were applied 5 times daily in a standardized class II cavity onto a plastic tooth. A precision-analysis scale was used to measure all of the following parameters before and after use: adhesive bottle, applicator, dosing plate, and plastic tooth. CEA was done by measuring the amount of material utilized/day, waste/day, efficacy, efficiency, average cost-effectiveness ratio (ACER), and incremental cost-effectiveness ratio (ICER). Data were analyzed using Kruskal-Wallis and Dunn's tests with Bonferroni correction at 0.05 significance level. CEA tested parameters were significantly different between groups $(p<0.001)$ except for ICER $(p=0.112)$. GP was the least effective (median $=0.062$ ), and SB was the least efficient (median $=0.366$ ). The highest and lowest ACER values were associated with TN (median cost $\approx$ USD 317) and SB (median $\operatorname{cost} \approx$ USD 317), respectively. ICER analysis reported an incremental cost for extra material utilized per milliliter of $\approx$ USD 208 for TN, USD 3.8 for GP, and USD -38 for OB, compared to SB. TN seems to be the most efficient and cost-effective dental adhesive.

Keywords: dental adhesive; cost-effectiveness; average cost-effectiveness ratio; incremental costeffectiveness ratio

\section{Introduction}

Dentistry is an expensive profession, and the expense of dental care is relatively high. Some of the expense related to dental care is the amount of waste that is produced as secondary outcome for providing treatment. This is the norm for multiple procedures including impression taking, direct restoration, and liner/base application. Decreasing waste and increasing the efficiency and/or efficacy of the procedure and utilized materials are paramount in maintaining a profitable career. Calculating the cost of this waste relative to the application benefit of the procedure is the basic principle of cost-effectiveness determination [1,2]. In the dental literature, there are multiple reports investigating the cost-effectiveness of restorative procedures. Chestnutt et al. [3] have investigated the costeffectiveness of fissure sealants application compared to fluoride varnish. Another study reported the cost-effectiveness of the repair of composite restorations in relation to total replacement [4].

The use of direct resin composite restorations is increasing due to the higher demand by patients for conservative esthetic solutions [5,6]. In addition, the shift to metal-free dental practices with phasing out of dental amalgam is leading to more focus on adhesive dentistry [7,8]. The technique sensitivity of older generation of dental adhesive led the manufacturers to develop new materials with simple use. Universal dental adhesives available in the market in 2011 are multipurpose bonding agents that can be applied either in etch-and-reins or in self-etch mode $[9,10]$. The composition of this type of adhesive plays an important role since it contains a functional monomer that ionically bonds to 
calcium in hydroxyapatites. One of the most used monomers is 10-methacryloyloxydecyl dihydrogen phosphate (MDP). It has mild etching properties, which enables the adhesive to be used with any etching technique. When the MDP monomer bonds to calcium, it forms a nonsoluble MDP-calcium salt, creating a strong and stable adhesion. The versatility of this type of adhesive allows the clinician to choose the most appropriate adhesion protocol for each type of procedure [10,11]. Furthermore, the presence of marginal gaps at the tooth structure-restoration interface resulting from polymerization shrinkage can have a deleterious effect on the longevity of the restoration [12]. The utilization of an efficient and predictable bonding technique can reduce the chance of marginal gap formation around resin composite restorations.

Although the conventional procedure for direct resin composite restoration placement is straight forward, it involves multiple steps and potential risk for waste. Among these, waste generated by unutilized adhesives can add up and progressively becomes a source of forfeiture that would be challenging to manage. Choosing a dental adhesive that can be used in an efficient manner is required in order to decrease this risk of accumulated loss. Some manufacturers have employed strategies that are aimed to decrease the resulting waste of the direct adhesive technique. The VivaPen device introduced with Tetric-N Bond Universal utilizes a replaceable micro applicator that can be used to directly apply the adhesive into the cavity, eliminating the need for a dosing plate and the accompanying waste. In addition, modifications of the geometry of the novel adhesive bottle introduced with G-Premio Bond, can restrict unnecessary and accidental dispensing of the dental adhesive.

To the authors' knowledge, there are no studies in the literature that investigated the waste and efficacy/efficiency of dental adhesives used for direct restorative applications. Thus, the aim of this study was to conduct a cost-effectiveness analysis (CEA) for four different universal dental adhesives. The null hypothesis was that there will be no difference between the different brands of adhesives in relation to each CEA parameter.

\section{Materials and Methods}

\subsection{Dental Adhesive Brands}

Four universal dental adhesives were used in the study: Single Bond Universal Adhesive (SB; 3 M ESPE, Dental Products, Saint Paul, MN, USA), Tetric N-Bond Universal VivaPen (TN; Ivoclar Vivadent, Zurich, Switzerland), OptiBond All-In-One (OB; Kerr Dental, Orange, CA, USA), and G-Premio Bond (GP; GC America, Alsip, IL, USA). Price (in USD), bottle capacity, and related information are included in (Table 1).

Table 1. Material information of the four adhesives used in the study.

\begin{tabular}{cccc}
\hline Dental Adhesive & Capacity $(\mathbf{m L})$ & Price ${ }^{*}$ (USD) & Price/mL (USD) \\
\hline SB & 5 & 82.3 & 16.5 \\
TN & 2 & 213.6 & 106.8 \\
OB & 5 & 83.9 & 16.8 \\
GP & 5 & 95.9 & 19.2 \\
\hline
\end{tabular}

SB: Single-Bond Universal, TN: Tetric N-Bond Universal VivaPen, OB: OptiBond All-In-One, GP: G-Premio Bond. * Includes the price for the adhesive bottle and the price for the applicators.

\subsection{Study Parameters}

The four dental adhesives were applied onto a standardized class II cavity preparation (measuring $2 \mathrm{~mm}$ pulpal floor depth, 3.5 gingival floor depth, and one-third intercuspal distance buccolingually) on an acrylic lower first molar tooth. Manufacturers' instructions were followed during the application process of each adhesive. During each application, the process of bonding agent application was done simulating a typical adhesive application for a class II composite restoration. For TN adhesive, the material was packed in a special delivery system, called VivaPen, that has a button to dispense the material. A disposable cannula with a brush was attached to the device, and three clicks were made to moisten the brush. The adhesive was applied and rubbed onto the cavity walls. For other adhesives, a 
single drop of the adhesive was dispensed into a dosing plate, after which a microbrush applicator was moistened with the adhesive and rubbed onto the cavity. The adhesive was applied in a single layer for all cavities. During all applications, the adhesive was subjected to a gentle stream of air for $5 \mathrm{~s}$ in order to evaporate the solvent.

All components of the process (adhesive bottle, applicator, dosing plate-not applicable for TN, and acrylic tooth) were weighed before and after each application using a precision analysis scale with a sensitivity of $0.0001 \mathrm{~g}$ (ZSA210, Scientech Inc., Boulder, CO, USA). The process was repeated five times per day for each dental adhesive, and multiple basic parameters were calculated, which are summarized in Table 2.

Table 2. Equations used for basic and cost-effectiveness analysis parameters.

1. Basic parameters:

a. Price per $\mathrm{mL}\left(\mathrm{P}_{\mathrm{mL}}\right)=\mathrm{P} / \mathrm{C}$

b. Application per $\mathrm{mL}\left(\mathrm{A}_{\mathrm{mL}}\right)=\Sigma \mathrm{A} / \mathrm{C}$

c. $\quad$ Price of each application $=\mathrm{P} / \Sigma \mathrm{A}$

d. $\quad \Sigma W=$ Waste in applicator + waste in dosing plate (not applicable for $\mathrm{TN}$ )

2. CEA parameters:

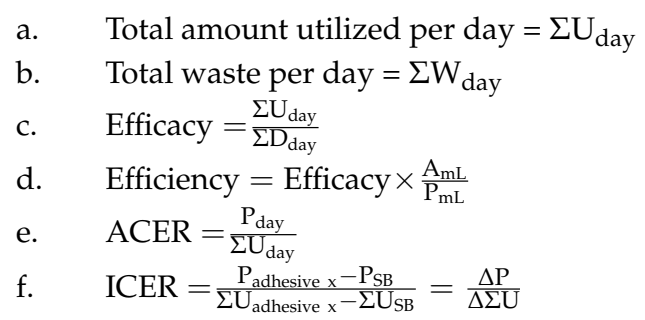

$\mathrm{P}=$ price, $\mathrm{C}=$ adhesive capacity, $\Sigma \mathrm{A}=$ total number of applications, $\Sigma \mathrm{W}=$ total waste, $\Sigma \mathrm{D}_{\text {day }}=$ total amount of adhesive dispensed per day.

\subsection{Cost-Effectiveness Analysis (CEA)}

The following parameters were used when determining the cost-effectiveness analysis: amount of material utilized per day, waste per day, efficacy, efficiency, average cost-effectiveness ratio (ACER), and incremental cost-effectiveness ratio (ICER). Efficacy reflects the benefit from the bonding procedure (amount of material utilized inside the cavity), while efficiency correlates the benefits to the cost (efficacy in relation to cost) [13]. The ACER calculates the average cost per effect, and the ICER reports on the difference in cost to the difference in effectiveness between one adhesive to a reference one [2]. All ICER comparisons were done using SB as a reference for comparison due to the abundant knowledge in the literature for its utilization in in vitro and in vivo investigations [14-16]. The CEA was calculated according to the equations found in Table 2.

\subsection{Statistical Analysis}

In order to conduct the statistical analysis, one day of application was considered as the unit of analysis for each adhesive. All CEA parameters were analyzed using the independent-samples Kruskal-Wallis test, followed by Dunn's test with the Bonferroni correction $(p<0.05)$ for the post hoc comparisons. All tests were conducted using the SPSS software (IBM SPSS Statistics, v25; IBM Corp., Armonk, NY, USA) for statistical analysis. ICER values were also compared between different adhesive brands. A negative and a positive ICER indicate better and worse cost-effectiveness compared to SB, respectively.

\section{Results}

The number of days and applications for each adhesive were as follow: SB adhesive lasted for 39 days with 192 applications; TN lasted for 37 days and 185 applications, OB for 20 days and 98 applications, and GP for 38 days and 188 applications. Descriptive statistics are illustrated in Table 3 and Figure 1. 
Table 3. Median values of the tested parameters.

\begin{tabular}{ccccccccc}
\hline $\begin{array}{c}\text { Dental } \\
\text { Adhesive }\end{array}$ & $\begin{array}{c}\text { Number of } \\
\text { Applications }\end{array}$ & $\begin{array}{c}\text { Applications/ } \\
\mathbf{m L}\end{array}$ & $\begin{array}{c}\text { Price/ } \\
\text { Application (USD) }\end{array}$ & $\begin{array}{c}\text { Total } \\
\text { Waste (mL) }\end{array}$ & Efficacy & Efficiency & ACER & ICER \\
\hline SB & 192 & 38.4 & 0.43 & 3.37 & 0.157 & 0.366 & 104.529 \\
TN & 185 & 92.5 & 1.15 & 1.14 & 0.337 & 3333.089 & 316.546 & 504.625 \\
OB & 98 & 19.6 & 0.86 & 1.5 & 0.081 & 26.811 & 206.104 & -76.070 \\
GP & 188 & 37.6 & 0.51 & 1.73 & 0.062 & 44.637 & 310.612 & 19.089 \\
\hline
\end{tabular}

(a)

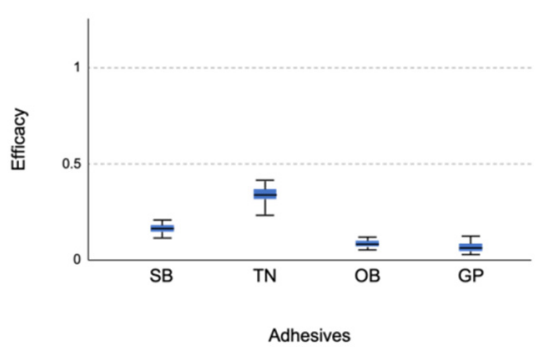

(c)

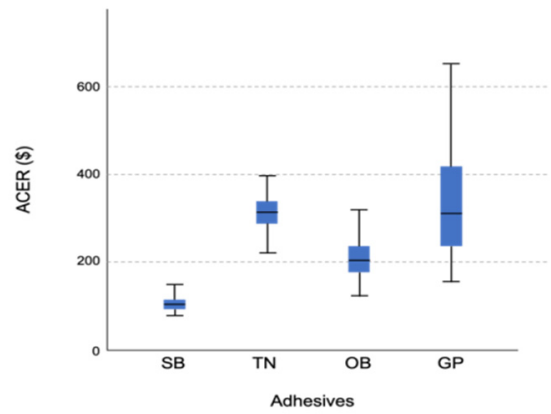

(b)

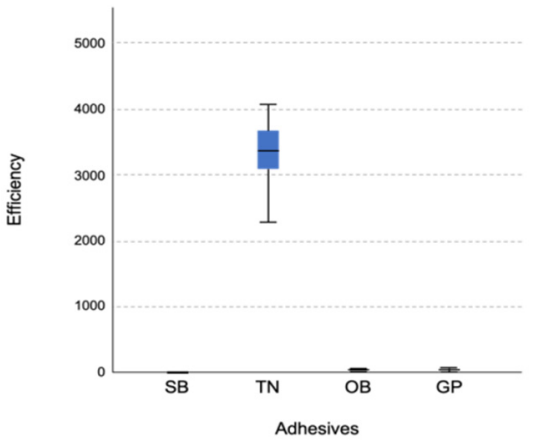

(d)

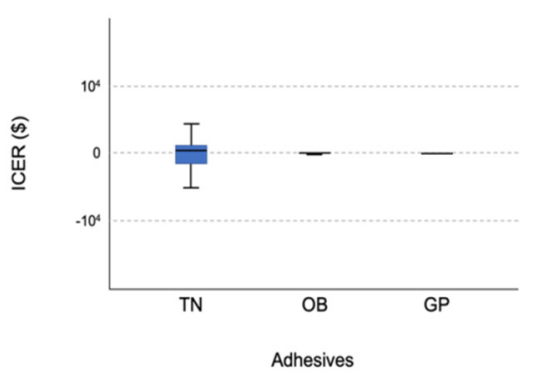

Figure 1. Whisker plots showing the study parameters: (a) efficacy, (b) efficiency, (c) ACER, and (d) ICER. SB: Single-Bond Universal, TN: Tetric N-Bond Universal VivaPen, OB: OptiBond All-In-One, GP: G-Premio Bond.

Assessing the assumption of normality with the Shapiro-Wilk test, it was significant in all of the test groups under each CEA parameter except the total amount of adhesive utilized per day. Accordingly, the nonparametric independent-samples Kruskal-Wallis test and Dunn's test with Bonferroni correction for post hoc comparison were used among all of the CEA parameters for days of application in order to standardize the statistical analysis method.

The independent-samples Kruskal-Wallis test was significant $(p<0.001)$ for all CEA parameters except ICER $(p=0.112)$. In terms of the total amount utilized per day, TN and $\mathrm{SB}$ groups were associated with the highest median $(0.019 \mathrm{~mL}(\mathrm{IQR}=0.02,0.02))$, and the least was in the GP group $(0.006 \mathrm{~mL}(\mathrm{IQR}=0.00,0.01))$. Post hoc comparison revealed significant differences among all of the groups except between the TN and SB groups and the $\mathrm{SB}$ and $\mathrm{OB}$ groups. For the total waste per day, the median amount of waste was found the least in the TN group $(0.031 \mathrm{~mL}(\mathrm{IQR}=0.03,0.03))$. The highest amount of waste was in the OB group $(0.758 \mathrm{~mL}(\mathrm{IQR}=0.69,0.82))$. Post hoc testing showed a significant difference between all of the groups except between the SB and OB groups.

With regards to efficacy and efficiency, TN was the most effective and efficient with median efficacy and efficiency value of 0.337 and 3333.089 , respectively. GP was the least effective with median value of 0.062 , and SB was the least efficient with median value of 0.366 . For efficacy post hoc comparison, there was a significant difference between all of 
the groups except between OB and GP. A similar finding was recorded for efficiency's post hoc analysis.

When ACER was defined as the price divided by the average amount used per day, SB group had a lower value (104.529) than the rest of the groups. The highest value was found in the TN group (316.546). The post hoc analysis revealed a significant difference among all of the tested adhesives except between the TN and GP. For the ICER parameter, OB had the lowest value (-76.070), followed by the GP (19.089) and the TN (504.0). However, the independent-samples Kruskal-Wallis test was not statistically significant $(p=0.112)$.

\section{Discussion}

The application of direct composite restorations is among the most commonly performed dental procedures. More than 5000 composite restorations are placed annually in a governmental dental school [17-19]. Waste during the application of the bonding technique can add up over time and contribute to the total expense of the operative procedure. Choosing a dental adhesive with less waste and more efficiency can maximize the benefit of the direct operative treatment. Thus, the aim of this study was to conduct a CEA analysis for commonly used universal dental adhesives for direct composite restorations. This would allow decision makers in governmental dental clinics and clinicians in private practices to decide on what type of adhesive to use to fit their budget and yet provide optimal effectiveness with minimal cost. However, we do not intend to provide recommendations on the actual clinical performance of the dental adhesives tested in this study since this was not the main focus of the current investigation. Readers should refer to other reports in the literature delineating actual performance of dental adhesives. The cost of adhesive should not be the sole factor for making a clinical decision. Other factors including ease of use, intended application, and the established clinical survival data should be considered.

The study also outlines a method used for CEA that can be adopted and modified by researchers for future studies. The CEA was done by testing six parameters: amount of material utilized and waste per day, efficacy, efficiency, ACER, and ICER. In order to compare the performance of each dental adhesive during daily practice and use, three relative parameters (price per $\mathrm{mL}$, application per $\mathrm{mL}$, and price per application) were utilized. We did not depend on the absolute milliliter figure of the bottle since the capacity of each dental adhesive bottle was different. Instead, the price per milliliter was used for all CEA comparisons. The null hypothesis was rejected in part as there was significant difference in all of the CEA parameters except the ICER.

Dental restorative procedures can produce a significant amount of material waste. One drop of adhesive for example can generate a lot of waste since there is always material left in the dosing plate and the applicator. Hence, with more applications, the cumulative waste associated with the adhesive bottle increases. The cumulative total waste generated from each adhesive tested in this study is illustrated in Figure 2. The cumulative waste of the $\mathrm{OB}$ adhesive was less than $50 \%$ of the bottle capacity $(5 \mathrm{~mL})$ and half of that associated with the SB adhesive. However, the SB adhesive provided nearly twice the number of applications as the OB adhesive did. Despite the fact that the SB adhesive provided the most applications (192), the cumulative waste associated with the additional number of applications almost reached $67.4 \%$ of the bottle capacity $(5 \mathrm{~mL})$. In comparison to other adhesives, the GP adhesive reported relatively low cumulative waste, with $34.6 \%$ of the bottle capacity $(5 \mathrm{~mL})$. The TN adhesive demonstrated nearly $57 \%$ cumulative waste of the VivaPen capacity $(2 \mathrm{~mL})$, translating into better overall efficiency. 


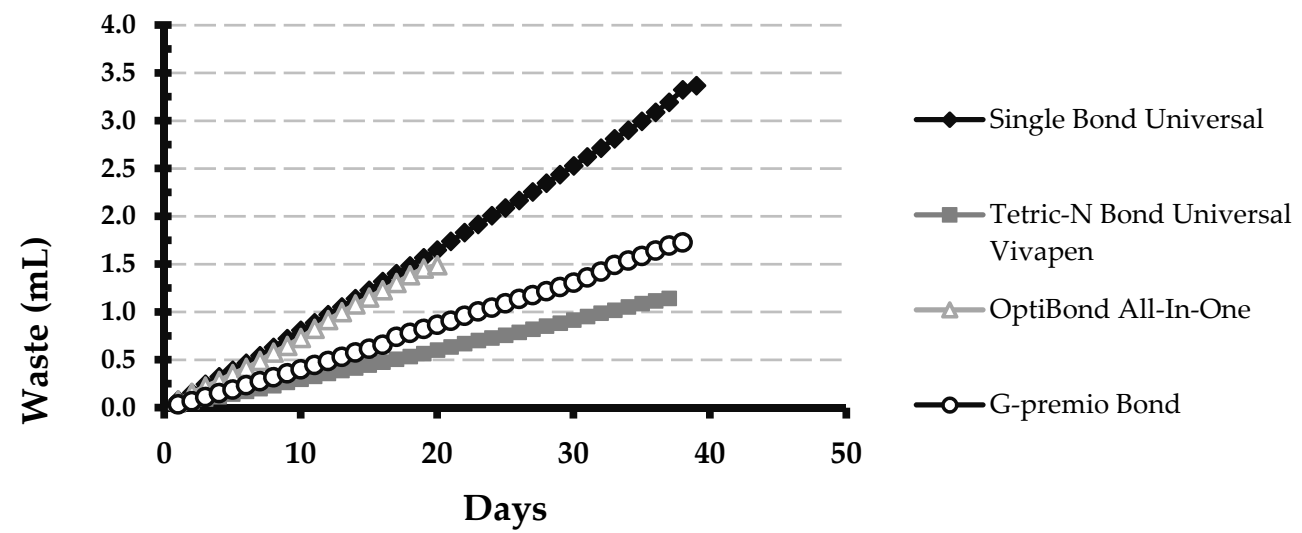

Figure 2. Cumulative total waste per day for the four adhesives used in this study.

Some manufacturers have hinted towards specific engineering choices in their products, such as the VivaPen delivery system used in TN. In this delivery system, the adhesive is packed in a pen-like device with a disposable brush cannula that allows for a direct application onto the tooth. According to the manufacturer, this delivery system can provide three times more applications per milliliter compared to conventional bottles with minimal waste optimizing efficiency. Another example of waste-reducing strategy is reported with GP adhesive, the manufacturer fitted the bottle with a silicone cover and a thin nozzle to enable more precise drop dispensing as commercially promoted. The result of this study showed that three clicks with the TN in VivaPen packaging and one drop of the SB adhesive were associated with the highest amount of material utilized inside the cavity and were enough to completely wet the walls. The OB adhesive demonstrated moderate amounts of material applied close to the TN and SB groups, and a single drop was actually enough to wet the cavity; however, this adhesive lasted only for 20 days with 98 applications and was associated with the highest amount of waste in the applicator and the dosing plate, even though the capacity of the bottle was comparable to that of the other adhesives. The opening of the $\mathrm{OB}$ adhesive bottle has a wide lumen with no integrated features to control the amount of adhesive dispensed, which allowed two drops to come out of the bottle on six occasions. In addition, remnants from the adhesive material accumulated around the opening of the bottle. Although GP was associated with the least amount of waste, a single drop of this adhesive failed to completely wet the cavity walls on many occasions with the lowest median amount of material utilized. This might indicate the need for two drops of GP adhesive in order to completely wet the class II cavity for composite restoration. This is reflected on the low efficacy score for the GP adhesive. The TN group had the highest values for efficacy (0.337) and efficiency (3333.089) since it had the highest values for utilization of the adhesive compared to waste. This supports the claim that this method of delivery would decrease the amount of waste in relation to cost, providing high efficiency with 185 applications, close to what the manufacturer claims (190 for one VivaPen). Although the SB adhesive had the second highest value for efficacy, it was found to be the least efficient when correlated to cost (SB: USD 16.5/1 mL, TN: USD 106.8/1 mL). This is explained by the relatively high amount of waste associated with this adhesive.

The main goal of CEA is to measure the trade-off between different resources and the outcome obtained [2]. ACER and ICER are some of the common methods used in CEA and play an important role in the assessment. However, both have different purposes and estimate different parameters. ACER for instance, measures the overall efficiency (cost to outcome) and provides an intuitive meaning about cost-effectiveness of one approach with a fixed budget independent of other options [2]. Nevertheless, sometimes it can be misleading and hide the high price of additional outcome gained per incremental cost. It basically distributes the cost over all subjects assuming all of them to have the same cost [1]. This can potentially lead clinicians to choose a less cost-effective approach. On the other hand, ICER reports on the incremental efficiency, which is the ratio of extra cost per 
extra effect compared to a common standard. Thus, it estimates whether the trade-off is cost-effective or not by looking into the true cost of additional gain in outcome [20-22].

In days of applications, the value of the ACER can estimate the potential increase in the price of the adhesive due to material waste. For example, the price of the SB and OB adhesives was USD 82 and USD 83.9, respectively. If we factor in the amount of waste associated with the bonding procedure, the actual cost of the adhesives will be USD 103 for the SB and USD 206 for the OB in order to utilize the wholeness of the material inside the bottle and not merely accepting that the material will be wasted. On the other hand, the ACER values for the TN and GP adhesives were the highest, costing USD 317 and USD 311, respectively.

Utilizing the ICER data, buying an extra amount of material utilized per milliliter was USD 208 for the TN adhesive and USD 3.8 for the GP. The incremental cost of the OB adhesive was USD - 38. In general, positive and negative ICER values indicate an increase and a decrease in benefit, respectively. In our study, however, the primary parameter of concern was the price, and a negative value would actually indicate more cost-effectiveness (benefit). In our findings, we found that the ICER value of $O B$ value was negative; however, there was no significant difference among the tested adhesives based on statistical analysis. This can be explained by the high amount of waste associated with $\mathrm{OB}$ adhesive and the low efficacy of the GP, both compared to the TN.

In this study, we presented several parameters that dental clinicians might want to look into and use as a guide for purchasing the most cost-effective adhesive that would fit their budgets. Taking into consideration all of the CEA and relative parameters, the TN adhesive seems to be the most cost-effective. Although the TN adhesive was associated with the highest ACER cost, compared to the three other adhesives with a capacity of $5 \mathrm{~mL}$, $\mathrm{TN}$ was the most effective and efficient with a capacity of only $2 \mathrm{~mL}$. In addition, there was no difference in the incremental cost associated with this adhesive. The fact that the TN adhesive can be applied directly onto the tooth eliminates the additional cost and waste associated with purchasing microbrush applicators and dosing plates required while using adhesives delivered in conventional bottles.

Similar to other in vitro studies, this investigation has some limitations. First, the study was not conducted in a clinical setting in which variable sizes and geometries of the cavities might affect the data. However, a laboratory approach was chosen in order to maintain low variability in the relevant factors, including the utilized cavity and the other conditions associated with the procedure. In addition, one of the major drawbacks of the ICER approach in this study was the use of one certain adhesive (the SB adhesive) as reference for comparison. Using another adhesive as a reference can potentially change the results and the data interpretation [20]. Moreover, we were not able to assess the incremental cost of the reference adhesive. However, SB is readily available in the market and it was used in multiple investigations previously [14-16]. Lastly, there was some difficulty in standardizing the applied force by which the adhesive bottles were squeezed in case of the SB, OB, and GP adhesives. This might have caused some variability in the amount of material dispensed from each adhesive on different occasions. However, one operator conducted the whole study in order to minimize the potential error.

Taking all of the CEA parameters into consideration, TN available in VivaPen seems to be the most efficient and cost-effective adhesive. Although the GP has an integrated feature to control the dispensing of the material, the outcome was not as efficient. SB adhesive was the least efficient when the performance was related to its price. The bottle of the OB adhesive has no features to control the amount of the material dispensed, which made this adhesive the least efficient with low number of applications.

Author Contributions: Conceptualization: H.M.N.; methodology: A.A.B. and H.M.N.; formal analysis: A.A.B. and H.M.N.; investigation: A.A.B.; resources: H.M.N.; data curation: A.A.B. and H.M.N.; writing-original draft preparation: A.A.B.; writing-review and editing: H.M.N.; visualization: A.A.B.; funding acquisition: H.M.N. All authors have read and agreed to the published version of the manuscript. 
Funding: This project was funded by Deanship of Scientific Research (DSR), King Abdulaziz University, Jeddah, Saudi Arabia, under grant No. (DF-049-165-1441). The authors gratefully acknowledge DSR technical and financial support.

Institutional Review Board Statement: Not applicable.

Informed Consent Statement: Not applicable.

Data Availability Statement: The data presented in this study are available on request from the corresponding author.

Conflicts of Interest: The authors declare no conflict of interest. VivaPen adhesive and some steps of the methodology were supplied by the local agent of Ivoclar Vivadent.

$\begin{array}{ll}\text { Abbreviations } \\ \text { CEA } & \text { Cost-effectiveness analysis } \\ \text { SB } & \text { Single-Bond Universal } \\ \text { TN } & \text { Tetric N-Bond Universal VivaPen } \\ \text { OB } & \text { OptiBond All-In-One } \\ \text { GP } & \text { G-Premio Bond } \\ \text { ACER } & \text { Average cost-effectiveness ratio } \\ \text { ICER } & \text { Incremental cost-effectiveness ratio } \\ \text { IQR } & \text { Interquartile range }\end{array}$

\section{References}

1. Hershey, J.C.; Asch, D.A.; Jepson, C.; Baron, J.; Ubel, P.A. Incremental and average cost-effectiveness ratios: Will physicians make a distinction? Risk Anal. 2003, 23, 81-89. [CrossRef]

2. Hoch, J.S.; Dewa, C.S. A clinician's guide to correct cost-effectiveness analysis: Think incremental not average. Can. J. Psychiatry 2008, 53, 267-274. [CrossRef]

3. Chestnutt, I.G.; Hutchings, S.; Playle, R.; Morgan-Trimmer, S.; Fitzsimmons, D.; Aawar, N.; Angel, L.; Derrick, S.; Drew, C.; Hoddell, C.; et al. Seal or Varnish? A randomised controlled trial to determine the relative cost and effectiveness of pit and fissure sealant and fluoride varnish in preventing dental decay. Health Technol. Assess. 2017, 21, 1-256. [CrossRef]

4. Kanzow, P.; Wiegand, A.; Schwendicke, F. Cost-effectiveness of repairing versus replacing composite or amalgam restorations. J. Dent. 2016, 54, 41-47. [CrossRef]

5. Demarco, F.F.; Correa, M.B.; Cenci, M.S.; Moraes, R.R.; Opdam, N.J. Longevity of posterior composite restorations: Not only a matter of materials. Dent. Mater. 2012, 28, 87-101. [CrossRef]

6. Jadhav, S.; Hegde, V.; Aher, G.; Fajandar, N. Influence of light curing units on failure of directcomposite restorations. J. Conserv. Dent. 2011, 14, 225-227. [CrossRef]

7. Domejean, S.; Leger, S.; Maltrait, M.; Espelid, I.; Tveit, A.B.; Tubert-Jeannin, S. Changes in Occlusal Caries Lesion Management in France from 2002 to 2012: A Persistent Gap between Evidence and Clinical Practice. Caries Res. 2015, 49, 408-416. [CrossRef]

8. Lynch, C.D.; McConnell, R.J.; Wilson, N.H. Trends in the placement of posterior composites in dental schools. J. Dent. Educ. 2007, 71, 430-434. [CrossRef]

9. Wagner, A.; Wendler, M.; Petschelt, A.; Belli, R.; Lohbauer, U. Bonding performance of universal adhesives in different etching modes. J. Dent. 2014, 42, 800-807. [CrossRef]

10. Scotti, N.; Cavalli, G.; Gagliani, M.; Breschi, L. New adhesives and bonding techniques. Why and when? Int. J. Esthet. Dent. 2017, 12, 524-535.

11. Sofan, E.; Sofan, A.; Palaia, G.; Tenore, G.; Romeo, U.; Migliau, G. Classification review of dental adhesive systems: From the IV generation to the universal type. Ann. Stomatol. 2017, 8, 1-17.

12. Lo Giudice, G.; Cicciù, M.; Cervino, G.; Lizio, A.; Visco, A.M. Flowable resin and marginal gap on tooth third medial cavity involving enamel and radicular cementum: A SEM evaluation of two restoration techniques. Indian J. Dent. Res. 2012, 23, 763-769.

13. Creugers, N.H.J.; Kayser, A.F. A Method to Compare Cost-Effectiveness of Dental Treatments-Adhesive Bridges Compared to Conventional Bridges. Community Dent. Oral Epidemiol. 1992, 20, 280-283. [CrossRef]

14. Balkaya, H.; Arslan, S.; Pala, K. A randomized, prospective clinical study evaluating effectiveness of a bulk-fill composite resin, a conventional composite resin and a reinforced glass ionomer in Class II cavities: One-year results. J. Appl. Oral Sci. 2019, 27. [CrossRef]

15. Frattes, F.C.; Augusto, M.G.; Torres, C.R.G.; Pucci, C.R.; Borges, A.B. Bond Strength to Eroded Enamel and Dentin Using a Universal Adhesive System. J. Adhes. Dent. 2017, 19, 121-127.

16. Yao, C.; Yang, H.; Yu, J.; Zhang, L.; Zhu, Y.; Huang, C. High Bond Durability of Universal Adhesives on Glass Ceramics Facilitated by Silane Pretreatment. Oper. Dent. 2018, 43, 602-612. [CrossRef] 
17. Bahannan, S.A.; Eltelety, S.M.; Hassan, M.H.; Ibrahim, S.S.; Amer, H.A.; El Meligy, O.A.; Al-Johani, K.A.; Kayal, R.A.; Mokeem, A.A.; Qutob, A.F.; et al. Oral and Dental Health Status among Adolescents with Limited Access to Dental Care Services in Jeddah. Dent. J. 2018, 6, 15. [CrossRef]

18. Al Agili, D.E. A systematic review of population-based dental caries studies among children in Saudi Arabia. Saudi Dent. J. 2013, 25, 3-11. [CrossRef]

19. Al-Meedani, L.A.; Al-Dlaigan, Y.H. Prevalence of dental caries and associated social risk factors among preschool children in Riyadh, Saudi Arabia. Pak. J. Med. Sci. 2016, 32, 452-456. [CrossRef]

20. Bang, H.; Zhao, H. Average cost-effectiveness ratio with censored data. J. Biopharm. Stat. 2012, 22, 401-415. [CrossRef]

21. Laska, E.M.; Meisner, M.; Siegel, C.; Stinnett, A.A. Ratio-based and net benefit-based approaches to health care resource allocation: Proofs of optimality and equivalence. Health Econ. 1999, 8, 171-174. [CrossRef]

22. Bang, H.; Zhao, H. Cost-effectiveness analysis: A proposal of new reporting standards in statistical analysis. J. Biopharm. Stat. 2014, 24, 443-460. [CrossRef] 